\title{
Efficient phase contrast imaging in STEM using a pixelated detector. Part 1: Experimental demonstration at atomic resolution
}

\author{
Timothy J. Pennycook ${ }^{\mathrm{a}, \mathrm{b}, *}$, Andrew R. Lupinic ${ }^{\mathrm{c}}$, Hao Yang ${ }^{\mathrm{b}}$, Matthew F. Murfitt ${ }^{\mathrm{d}, 1}$, Lewys Jones ${ }^{\mathrm{b}}$, Peter D. Nellist , $^{\mathrm{b}, \mathrm{a}}$ \\ ${ }^{a}$ EPSRC SuperSTEM Facility, Daresbury Laboratory, Warrington, WA4 4AD, UK \\ ${ }^{b}$ Department of Materials, University of Oxford, Parks Road, Oxford OX1 3PH, UK \\ ${ }^{c}$ Oak Ridge National Laboratory, Materials Science and Technology Division, Oak Ridge, TN 37830, USA \\ ${ }^{d}$ Nion Co., 1102 8th St., Kirkland, WA 98033, USA
}

\begin{abstract}
We demonstrate a method to achieve high efficiency phase contrast imaging in aberration corrected scanning transmission electron microscopy (STEM) with a pixelated detector. The pixelated detector is used to record the ronchigram as a function of probe position and then analysed with ptychography. Ptychography has previously been used to provide super-resolution beyond the diffraction limit of the optics, alongside numerically correcting for spherical aberration. Here we rely on a hardware aberration corrector to eliminate aberrations, but use the pixelated detector data set to utilise the largest possible volume of Fourier space to create high efficiency phase contrast images. The use of ptychography to diagnose the effects of chromatic aberration is also demonstrated. Finally, the four dimensional dataset is used to compare different bright field detector configurations from the same scan for a sample of bilayer graphene. Our method of high efficiency ptychography produces the clearest images, while annular bright field produces almost no contrast for an in-focus aberration-corrected probe.
\end{abstract}

Keywords: STEM, Pixelated detectors, Ptycography, phase contrast, chromatic aberrations, DPC, ABF

\section{Introduction}

For imaging beam sensitive weak phase biological specimen, Henderson et al. 11 pointed out that "For X-rays, the amount of damage per useful elastic scattering event is several hundred times greater than for electrons at all wavelengths and energies". Single elastic scattering conditions for electron scattering correspond to the weak-phase object approximation. For this and many other reasons, phase contrast imaging in the transmission electron microscope (TEM) continues to play a crucial role in the imaging of many different sample types.

Conventionally in high-resolution (HR-)TEM, an approximate phase plate is formed using the inherent aberrations of rotationally symmetric electron lenses. The inherent positive spherical aberration can be used along with an optimally chosen defocus in the so-called Scherzer condition [2]. The development of hardware aberration correctors allows further refinement of the effective phase plate, and indeed the use of negative spherical aberration phasecontrast imaging [3]. Alongside this, there continues to be considerable interest in the development of physical phaseplate devices for TEM [4].

Through the principle of reciprocity [5, 6, 7] phase contrast imaging can be performed using an axial detector in

\footnotetext{
*Corresponding author. E-mail: tpennycook@gmail.com (T.J. Pennycook).

${ }^{1}$ Present address: 511 E Roy St \#315, Seattle, WA 98102, USA.
}

the scanning TEM (STEM) and making use of aberrations to form a phase plate. Because the angles subtended by the detector are necessarily smaller than the beam convergence angle, the efficiency of this mode of imaging is poor, with many electrons not being collected by the detector. Furthermore, a key strength of STEM is the ability to record the annular dark-field (ADF) signal and analytical signals such as energy-dispersive X-ray and electron energy-loss spectroscopy (EELS). Because all these latter imaging modes are incoherent, the optimal STEM probe is the smallest, most intense one, which is achieved by nulling all the aberrations as far as possible, thereby leading to poor phase contrast in the conventional bright-field mode.

In STEM, however, a wide range of detector geometries are available, and Harold Rose has proposed and explored a number of these. Two successful examples are annular bright field (ABF) and differential phase contrast (DPC) imaging. The use of ABF was first suggested by Rose in 1973 [8] to enhance the phase contrast in STEM. The detector system consists of an ABF detector to collect the bright field signal and a full bright field (BF) detector beneath to collect the remaining bright field signal, and the image contrast is enhanced by taking the difference between the two detector signals. Although it was shown that this technique requires a dose 4 times higher than TEM, it offers an image containing fewer artefacts and finer resolved object details. Due to its sensitivity to 
imaging light elements using aberration corrected microscopes, ABF has become an increasingly popular technique in the past few years 9 . In the context of resolving light elements in crystals also containing heavy elements, the contrast is ascribed to the effects of channeling [10] and therefore requires multiple scattering.

Differential phase contrast (DPC) was first proposed by Dekkers and DeLang [11, and uses a detector consisting of two half discs with the bisection perpendicular to the scanning direction. It was shown on theoretical grounds 12 that by taking the difference signal between the pair of sectors, DPC image contrast represents the gradient of the object potential along the direction of scan. To enable contrast transfer in two dimensions, a four quadrant detector geometry was proposed by Rose in 1977 [13]. DPC was subsequently applied to the studies of magnetic domain structures by Chapman et al., [14] and an annular DPC with eight segments was further introduced for visualising longer range magnetic fields [15]. Using an aberration corrected microscope, imaging of electrostatic fields at atomic resolution [16] has also been achieved with DPC.

The key to designing detector geometries to obtain the greatest signal, as Rose pointed out [13, is to collect the constructive and destructive interferences in the convergent beam electron diffraction (CBED) pattern by separate detectors. A method of improving the resolution of the STEM was developed by Rodenburg et al. [17. It was shown that with a sufficiently redundant data set of coherent microdifffraction patterns collected as a function of probe position, it is possible to recover the phase information of the transmission function and remove the effects of lens aberrations. Using this technique Nellist et al. were able to resolve silicon atoms spaced $1.36 \AA$ apart on a $100 \mathrm{kV}$ STEM instrument with no aberration corrector, a conventional point resolution of $4.2 \AA$ and a conventional information limit of $3.3 \AA 18$.

Although there continues to be progress in superresolution techniques using ptychography in electron microscopy [19, 20, the successful production of hardware based aberration correctors and their widespread use in high resolution TEM and STEM now provides a direct route for enhanced resolution and sensitivity for incoherent imaging modes such as ADF and EELS. There have also been been recent advances in both X-ray and light microscopy [21, 22, 23, 24, 25] using ptchography. However, ptychography still holds great potential for use in high resolution transmission electron microscopy. Rather than using the method to improve spatial resolution, in the present paper we describe the use of ptychography with a pixelated detector to improve the efficiency of STEM phase contrast imaging and to diagnose chromatic aberrations.

With ptychography we can use the entire bright field disk to form phase contrast images. However, in order to counteract lens aberrations, earlier work with ptychography focused on using only a relatively small region of the detector plane for each image spatial frequency [17. Here we instead utilise all available regions of the detector plane to perform phase contrast imaging with the maximum possible efficiency in STEM. Furthermore, by selectively collecting phase and amplitude information only from regions of the detector plane where the wavefunctions are transferred for each spatial frequency we reject noise, resulting in clearer, less noisy images. This efficiency means that the technique could be very promising for use with beam sensitive materials where high speed pixelated detectors should allow image contrast to be retained even with very short dwell times.

Particularly at low voltages, chromatic aberrations are often the limiting form of aberration in modern spherical aberration corrected STEM instruments and lead to partial temporal coherence. Moreover, following the recent development of hardware which in theory is capable of countering chromatic aberrations [26], a method to measure the effects of partial temporal coherence directly from image data is desirable. Nellist et al. 27] showed theoretically how ptychography can be used to diagnose partial temporal coherence. Here we provide the first experimental demonstration of this method and use it to measure the energy-spread of the incident beam.

Using a pixelated detector to record the variation of the ronchigram as a function of probe position provides the ability to choose the bright field detector configurations after acquisition. To produce a given detector geometry is simply a matter of choosing which regions of the bright field disk to integrate over at each probe position. One can also perform mathematical operations between different regions of the bright field disk to, for instance, produce a DPC image. This ability to produce multiple types of bright field images from the same dataset allows us to directly compare different bright field configurations. We compare several popular bright field detector configurations in use today to phase contrast images formed with ptychography. Compared to images formed with conventional BF, DPC, and ABF configurations, maximum efficiency ptychography does indeed produces the clearest images, with the highest signal to noise ratio. Thus this technique may present a very promising method to image light elements in STEM. ABF images of graphene show essentially no contrast, as indeed should be expected for a centrosymmetric detector configuration in the absence of significant aberrations.

\section{Extraction of phase and amplitude}

\subsection{Theoretical background}

The intensity in a ronchigram is the modulus squared of the wavefunction of the fast electrons at the back focal plane, $\left|\Psi_{f}\left(\mathbf{K}_{f}, \mathbf{R}_{p}\right)\right|^{2}$, where $\mathbf{K}_{f}$ denotes the angle or position on the ronchigram and $\mathbf{R}_{p}$ the probe position. Fourier transforming with respect to probe position one obtains

$$
G\left(\mathbf{K}_{f}, \mathbf{Q}_{p}\right)=\int\left|\Psi_{f}\left(\mathbf{K}_{f}, \mathbf{R}_{p}\right)\right|^{2} \exp \left(2 \pi i \mathbf{R}_{p} \cdot \mathbf{Q}_{p}\right) d \mathbf{R}_{p}
$$


where $\mathbf{Q}_{p}$ is the spatial frequency. Rodenburg et al. 17. showed that for a weak phase object this can be rewritten as

$$
\begin{aligned}
G\left(\mathbf{K}_{f}, \mathbf{Q}_{p}\right)= & \left|A\left(\mathbf{K}_{f}\right)\right|^{2} \delta\left(\mathbf{Q}_{p}\right) \\
& +A\left(\mathbf{K}_{f}\right) A^{*}\left(\mathbf{K}_{f}+\mathbf{Q}_{p}\right) \Psi_{s}^{*}\left(-\mathbf{Q}_{p}\right) \\
& +A^{*}\left(\mathbf{K}_{f}\right) A\left(\mathbf{K}_{f}-\mathbf{Q}_{p}\right) \Psi_{s}\left(\mathbf{Q}_{p}\right),
\end{aligned}
$$

where $\Psi_{s}\left(\mathbf{Q}_{p}\right)$ is the Fourier transform of the object transmission function at a spatial frequency of $\mathbf{Q}_{p}$ and would be the scattered wavefunction under conditions of planewave illumination, and $A\left(\mathbf{K}_{f}\right)=a\left(\mathbf{K}_{f}\right) \exp \left[i \chi\left(\mathbf{K}_{f}\right)\right]$, the aperture function, describes the modulus and phase of the transfer function of the objective lens. $a\left(\mathbf{K}_{f}\right)$ is the modulus of $A\left(\mathbf{K}_{f}\right)$ and is one inside the objective aperture and zero outside the aperture. The phase of the aperture function, $\chi\left(\mathbf{K}_{f}\right)$, is determined by the lens aberrations. The terms $A\left(\mathbf{K}_{f} \pm \mathbf{Q}_{p}\right)$ represent scattered disks centred at $\mathbf{K}_{f}=\mathbf{Q}_{p}$. The multiplied pairs of aperture functions in the second and third terms on the right hand side of equation 2 act as logical ANDs: each pair has nonzero amplitude only where the zero order and scattered disk overlap.

Rodenburg et al. also showed that in the weak phase approximation

$$
\Psi_{s}\left(\mathbf{Q}_{p}\right)=-\Psi_{s}^{*}\left(-\mathbf{Q}_{p}\right) .
$$

This implies the complex values of $\Psi_{s}\left(\mathbf{Q}_{p}\right)$ and $\Psi_{s}^{*}\left(-\mathbf{Q}_{p}\right)$ have the same amplitude but are are $\pi$ radians out of phase. Therefore, in the absence of aberrations, the total phase of the second and third terms on the right hand side of equation 2 will also be $\pi$ out of phase. In triple overlap regions, where the two scattered disks overlap inside the zero order disk, the last two terms will cancel and no wavefunction transfer will occur. However in double overlap regions, where only one scattered disk overlaps with the zero order disk, the wavefunctions transfer (as illustrated in Figure 11). Furthermore, because the wavefunctions in equation 2 are not contained within modulus squares, it is possible to access their phase and amplitude.

Previously, this means of accessing the phase and amplitude of $\Psi_{s}\left(\mathbf{Q}_{p}\right)$ was used to achieve an improved resolution [17. Points along the line $\mathbf{K}_{f}=\mathbf{Q}_{p} / 2$ in $G\left(\mathbf{K}_{f}, \mathbf{Q}_{p}\right)$ are midway between the centre of the zero order disk $\left(\mathbf{K}_{f}=\right.$ $0)$ and centre of the scattered disk $\left(\mathbf{K}_{f}=+\mathbf{Q}_{p}\right)$. Therefore because of the symmetry of the round aberrations, the spherical aberrations in $A\left(\mathbf{K}_{f}\right)$ and $A\left(\mathbf{K}_{f}-\mathbf{Q}_{p}\right)$ are the same at $\mathbf{K}_{f}=\mathbf{Q}_{p} / 2$. The spherical aberrations (and in fact all round aberrations) therefore cancel in $A\left(\mathbf{K}_{f}\right) A^{*}\left(\mathbf{K}_{f}-\right.$ $\left.\mathbf{Q}_{p}\right)$ thanks to the complex conjugate. Similarly, the spherical aberrations cancel in the expression $A\left(\mathbf{K}_{f}\right) A^{*}\left(\mathbf{K}_{f}+\right.$ $\left.\mathbf{Q}_{p}\right)$. Hence by extracting $\Psi_{s}\left(\mathbf{Q}_{p}\right)$ only at points satisfying $\mathbf{K}_{f}=\mathbf{Q}_{p} / 2$ in $G\left(\mathbf{K}_{f}, \mathbf{Q}_{p}\right)$ it is possible to determine the phase and amplitude of the scattered wavefunctions without the influence of round aberrations. It is then a simple matter of Fourier transforming to obtain a real space phase contrast image free of spherical aberration. Since the usual

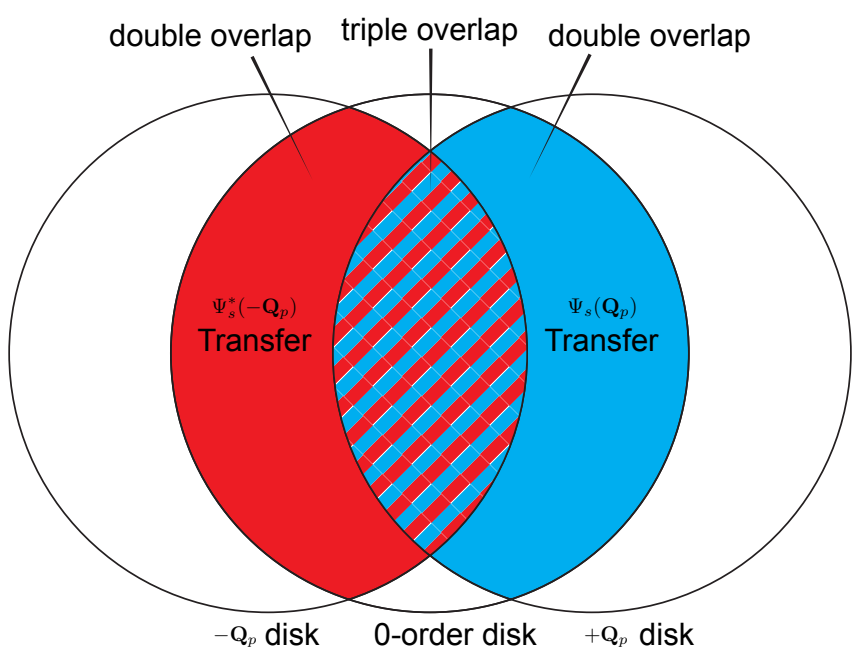

Figure 1: Schematic of $G\left(\mathbf{K}_{f}, \mathbf{Q}_{p}\right)$ for a fixed $\mathbf{Q}_{p}$ with a region of triple overlap. The lefthand disk is centred at $\mathbf{K}_{f}=-\mathbf{Q}_{p}$, the central disk at $\mathbf{K}_{f}=0$ and the righthand disk at $\mathbf{K}_{f}=\mathbf{Q}_{p}$. The lefthand double overlap region transfers $\Psi_{s}^{*}\left(-\mathbf{Q}_{p}\right)$, the righthand double overlap region transfers $\Psi_{s}\left(\mathbf{Q}_{p}\right)$. For a weak phase object, $\Psi_{s}\left(\mathbf{Q}_{p}\right)$ is the same as $\Psi_{s}^{*}\left(-\mathbf{Q}_{p}\right)$, except for a $\pi$ phase difference. In the triple overlap region, both wavefunctions are transferred, but because they are in antiphase, cancel each other out.

limit for a small axial detector to transfer a particular spatial frequency is that $\mathbf{Q}_{p}$ must be inside the aperture, this method allows spatial frequencies up to twice the usual limit to be transferred.

Although small residual aberrations may remain in a modern aberration corrected instrument, atomic resolution imaging is largely routine, and the limiting parameter is often beam induced damage rather than the spatial resolution of the microscope. Hence we have developed a means to use ptychography to achieve the maximum efficiency phase contrast imaging possible in STEM. Rather than using a small subset of points in $G\left(\mathbf{K}_{f}, \mathbf{Q}_{p}\right)$ to remove aberrations, we rely on the aberration corrector hardware to eliminate aberrations and instead use as much of the four dimensional volume of $G\left(\mathbf{K}_{f}, \mathbf{Q}_{p}\right)$ as possible to increase the amount of information gained per fast electron.

Instead of extracting the wavefunctions from just one point per spatial frequency, we extract the phase and amplitude from the entirety of each double overlap region for every frequency. For a given spatial frequency, $\mathbf{Q}_{p}$, we add $\pi$ to the phase of one of the double overlap regions. We also set the amplitudes outside the double overlap regions to zero because for a weak phase object only noise is contained outside the double overlap regions in the absence of aberrations. We then calculate the Fourier component for that spatial frequency by summing over all $\mathbf{K}_{f}$ to obtain the best statistics on the phase and amplitude of $\Psi_{s}\left(\mathbf{Q}_{p}\right)$. Once we have determined $\Psi_{s}\left(\mathbf{Q}_{p}\right)$ for all $\mathbf{Q}_{p}$, it is straightforward to form an image by Fourier transforming back to real space to form a high efficiency phase contrast image. 

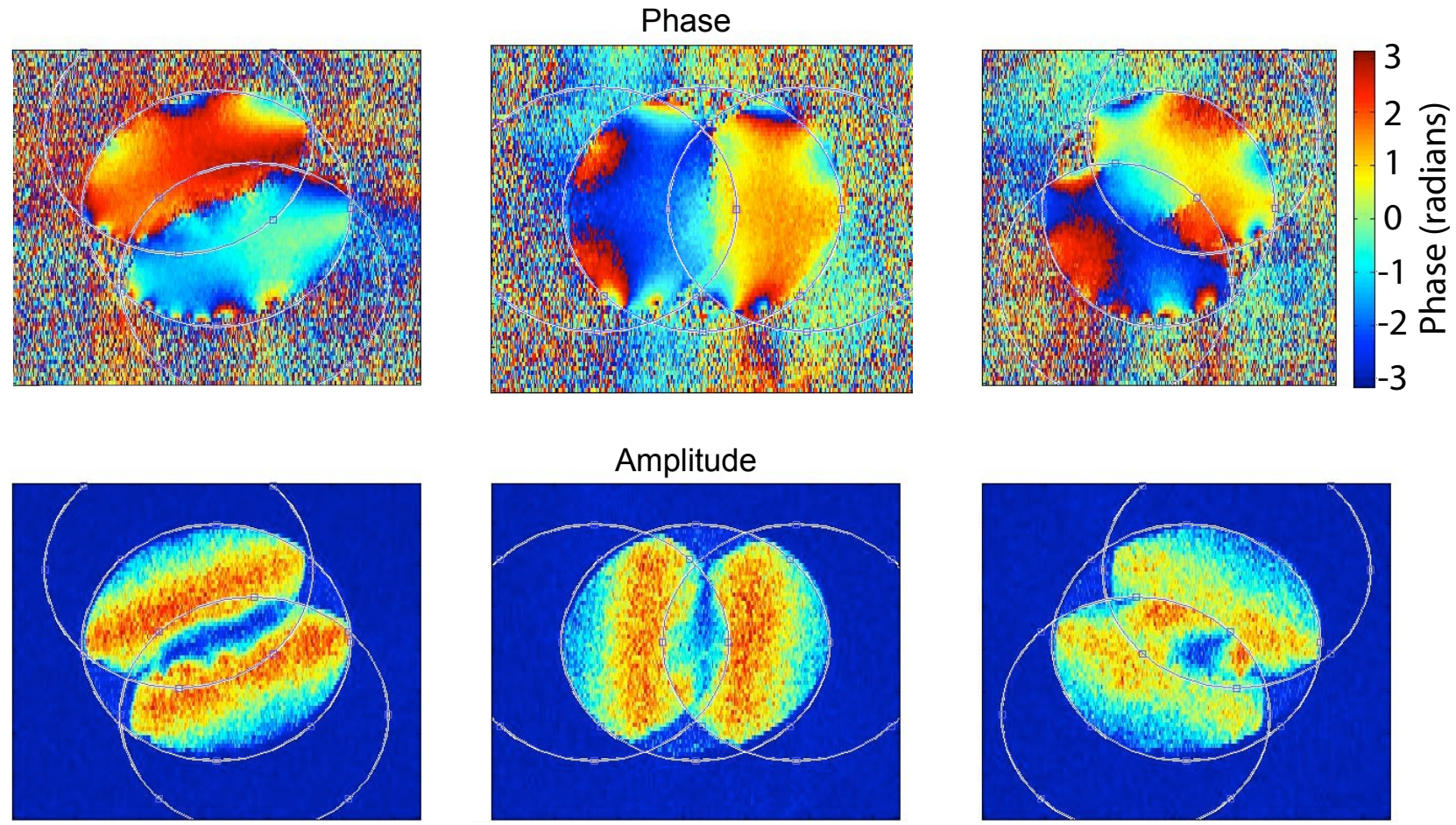

Figure 2: The phase and amplitude of $G\left(\mathbf{K}_{f}, \mathbf{Q}_{p}\right)$ as a function of $\mathbf{K}_{f}$ for three $\mathbf{Q}_{p}$ values with significant amplitude extracted from a four dimensional dataset from a sample of graphene. The white circles indicate the approximate positions of the zero order and scattered disks as a guide to the eye.

\subsection{Data acquisition}

A Nion CCD1010 camera was used on a Nion UltraSTEM $200[28$ to record a ronchigram at every probe position. The microscope was operated using a $60 \mathrm{kV}$ accelerating voltage and a 30 mrad convergence angle. The camera clock out was used to advance the scan each time the camera finishes a frame. This hardware synchronization allows the scan to advance at the same rate as the camera can record each ronchigram. The exception to this was at the end of each horizontal line of the scan, where two extra images were required to advance the scan to the next row of pixels. 8 times vertical binning was used to increase the frame rate of the camera to approximately 30 frames per second. This allowed us to record 64 by 64 probe position data sets, containing 4225 ronchigrams, in approximately three minutes. The high angle annular dark field (HAADF) intensity was recorded simultaneously at each probe position, providing a Z-contrast image for comparison.

\subsection{Analysis}

The ronchigram images were processed with an in house written Matlab code. The code first removes the extra ronchigram images that were recorded at the end of each row of the scan. The remaining images are then Fourier transformed with respect to probe position. The phase and amplitude of $G\left(\mathbf{K}_{f}, \mathbf{Q}_{p}\right)$ can then be displayed as a function of $\mathbf{K}_{f}$ for any desired $\mathbf{Q}_{p}$ present in the dataset. Examples of the phase and amplitude of $G\left(\mathbf{K}_{f}, \mathbf{Q}_{p}\right)$ extracted from a four dimensional dataset acquired from a sample of graphene are shown in Figure 2. Shown are the phase and amplitude of $G\left(\mathbf{K}_{f}, \mathbf{Q}_{p}\right)$ for values of $\mathbf{Q}_{p}$ which correspond to lattice spacings in the graphene sheet so that the data shows a significant amplitude. In an aberration-corrected instrument, it might be expected that the phase of $A\left(\mathbf{K}_{f}\right)$ is zero, and that therefore the phase of $G\left(\mathbf{K}_{f}, \mathbf{Q}_{p}\right)$ within the double overlap region is constant. The experimental data does, however, show phase variations in the double overlap region. There are both longer range variations and very localized variations at the aperture edge. The former we ascribe to residual aberrations from imperfect tuning of the corrector, and the latter to localized contamination on the aperture edge that is charging under electron irradiation. Despite these phase variations, it can be seen that the two double overlap regions for each of these $\mathbf{Q}_{p}$ are out of phase. The amplitudes can also be seen to be more or less equal for symmetric points in the pairs of double overlap regions. The amplitudes are also seen to be maximum along lines forming the perpendicular bisectors of the lines between $\mathbf{K}_{f}=0$ and $\mathbf{K}_{f}= \pm \mathbf{Q}_{p}$. This is due to chromatic aberration, as will be discussed in more detail later.

Once the relationship between $\mathbf{Q}_{p}$ and the position of the scattered disks is determined it is possible to auto- 


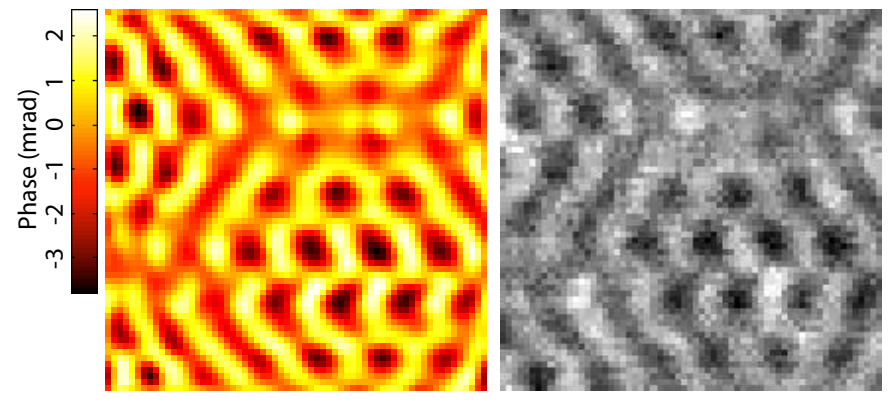

Figure 3: Maximum efficiency phase contrast (left) and HAADF (right) images of bilayer graphene. The phase contrast image was created by using ptycography to determine the phase and amplitude of all possible spatial frequencies, using all available double overlap regions. The HAADF image was acquired simultaneously with the $4 \mathrm{D}$ data set of ronchigrams used to construct the phase contrast image. The phase contrast image is clearly less noisy than the HAADF image, reflecting the efficiency and noise rejection of the method employed to construct it.

mate the selection of the double overlap regions. The code we have developed automatically selects each of the two double overlap regions for every $\mathbf{Q}_{p}$ available in the four dimensional dataset. For each spatial frequency, the phase of one of the two double overlap regions is shifted by $\pi$ to bring it into phase with the other region of double overlap. We denote this modified phase matrix as $\phi\left(\mathbf{K}_{f}, \mathbf{Q}_{p}\right)$. The amplitude is also modified such that it is zero outside the double overlap regions (except for $\mathbf{Q}_{p}=0$, to retain the correct DC level) in a matrix which we call $\alpha\left(\mathbf{K}_{f}, \mathbf{Q}_{p}\right)$. The sum

$$
S\left(\mathbf{Q}_{p}\right)=\sum_{\mathbf{K}_{f}} \alpha\left(\mathbf{K}_{f}, \mathbf{Q}_{p}\right) e^{i \phi\left(\mathbf{K}_{f}, \mathbf{Q}_{p}\right)}
$$

is then calculated. $S\left(\mathbf{Q}_{p}\right)$ is $G\left(\mathbf{K}_{f}, \mathbf{Q}_{p}\right)$ summed over the double overlap regions. $S\left(\mathbf{Q}_{p}\right)$ can be regarded as $\Psi_{s}\left(\mathbf{Q}_{p}\right)$ that has been Fourier filtered with a transfer function given by the area of the double overlap for that spatial frequency (see Yang et al. in part II of these papers). The inverse Fourier transform of $S\left(\mathbf{Q}_{p}\right)$ is then performed, interfering all the scattered beams to give the real space complex wavefunction, $\Psi_{s}\left(\mathbf{R}_{p}\right)$.

An example of such a phase contrast image of twisted bilayer graphene is shown in Figure 3 alongside a simultaneously acquired HAADF image. The improved signal to noise ratio of the phase contrast image is striking when compared to the HAADF image. Although only a small fraction of the incident probe is scattered out to high angles for the graphene sample, the HAADF detector is able to detect even single electrons [29], whereas this particular CCD camera is not cooled or optimized for low noise. As the data for the two images was acquired in parallel, they use the same dwell time, and hence the clarity of the phase contrast image reflects the efficiency and noise rejection of the method used to construct it. We emphasise that this efficiency is due to the extraction of phase and amplitude information from and only from all regions of Fourier space where the transfer of $\Psi_{s}\left(\mathbf{Q}_{p}\right)$ is expected for a weak phase object. Furthermore, because we also collect the ADF signal, we collect data from essentially all available angles, maximising the information gained per electron in the probe. We note that using the medium angle ADF (MAADF) signal would cover an even greater range of scattering angles and provide a greater signal to noise ratio for $\mathrm{ADF}$ imaging of graphene and other single or few layer materials. The MAADF signal was not however available simultaneously with the imaging mode used to record the ronchigrams at the time the present experiments were performed.

With the atomic number contrast of ADF images, having the ADF data alongside the phase contrast images significantly improves the interpretability of the datasets. For instance, in Figure 3 the HAADF image shows columns which are brighter where the atoms of the two graphene sheets align. Additionally, it is worth pointing out that by using all available frequencies in the construction of the phase contrast image, the method should be sensitive to light impurity atoms and molecules which are not so easily revealed by $\mathrm{ADF}$ imaging. The phase contrast in figure 3 is actually slightly lower than that expected from simulations (see Yang et al. in part II of these papers) and this will be the subject of future investigations.

\section{Chromatic aberrations}

Chromatic aberrations result from variations in the energy of the electrons in the electron probe, resulting in a spread of defocus values which can limit the resolution of a microscope. In STEM instruments equipped with spherical aberration correctors, chromatic aberration is often the factor limiting the maximum resolution. With recent development of a monochromator which should be capable of correcting chromatic aberrations in STEM [26] and chromatic aberration correctors [30, a method for measuring the effects of chromatic aberrations directly from the image data is desirable. The theoretical underpinnings of such a technique were first laid down by Nellist et al. [27, but the method had not previously been used to measure chromatic aberrations experimentally.

Chromatic aberrations cause the variation in the amplitude of $G\left(\mathbf{K}_{f}, \mathbf{Q}_{p}\right)$ across $\mathbf{K}_{f}$ for a given $\mathbf{Q}_{p}$ exemplified by the amplitude distributions shown in Figure 2. The fluctuations in defocus caused by partial coherence affect the phase of the aperture functions $A\left(\mathbf{K}_{f}\right)=a\left(\mathbf{K}_{f}\right) \exp \left[\chi\left(\mathbf{K}_{f}\right)\right]$. In the absence of non-round aberrations

$$
\chi\left(\mathbf{K}_{f}\right)=\pi C_{1,0} \lambda\left|\mathbf{K}_{f}\right|^{2}+\cdots,
$$

where $C_{1,0}$ is the defocus. As $\chi\left(\mathbf{K}_{f}\right)$ is radially symmetric, $A\left(\mathbf{K}_{f}\right)$ and $A\left(\mathbf{K}_{f} \pm \mathbf{Q}_{p}\right)$ will have the same phase at points that are equidistant from the centres of the zero order disk and the scattered disk for a given $\mathbf{Q}_{p}$. At these points, changes in phase due to defocus in $A\left(\mathbf{K}_{f}\right) A^{*}\left(\mathbf{K}_{f}+\mathbf{Q}_{p}\right)$ or $A^{*}\left(\mathbf{K}_{f}\right) A\left(\mathbf{K}_{f}-\mathbf{Q}_{p}\right)$ will cancel out because of the complex conjugations. The points that are equidistant from 
the centres of the two disks are therefore immune from variations in defocus, and form what are called achromatic lines in $G\left(\mathbf{K}_{f}, \mathbf{Q}_{p}\right)$.

As illustrated in Figure 4, the achromatic lines appear at the midpoint between the centres of the overlapping disks and extend perpendicularly to the vector linking their centre points. The effects of defocus fluctuation increase with increasing distance from the achromatic lines in $\mathbf{K}_{f}$ as the cancelation due to the complex conjugation diminishes moving in the direction perpendicular to the lines. This variation can be described as a four dimensional envelope function modulating the amplitude of the $G\left(\mathbf{K}_{f}, \mathbf{Q}_{p}\right)$ in the absence of any defocus spread, $G^{\text {ach }}\left(\mathbf{K}_{f}, \mathbf{Q}_{p}\right)$, as

$$
\begin{aligned}
G\left(\mathbf{K}_{f}, \mathbf{Q}_{p}\right) & \\
& =G^{\mathrm{ach}}\left(\mathbf{K}_{f}, \mathbf{Q}_{p}\right) \\
& \times \exp \left[-\frac{1}{2} \pi^{2} \lambda^{2} \Delta^{2}\left(2 \mathbf{K}_{f} \cdot \mathbf{Q}_{p}+\left|\mathbf{Q}_{p}\right|^{2}\right)^{2}\right]
\end{aligned}
$$

where $\Delta$ is the incoherent defocus spread [27]. Using this equation it is possible to determine $\Delta$ by fitting to the amplitude of $G\left(\mathbf{K}_{f}, \mathbf{Q}_{p}\right)$ at a $\mathbf{Q}_{p}$ with an amplitude function with a Gaussian profile perpendicular to the achromatic line for a value of $Q_{p}$ where $G\left(\mathbf{K}_{f}, \mathbf{Q}_{p}\right)$ has a significant amplitude. Using Matlab to perform the fit, we find $\Delta=2.8 \mathrm{~nm} . \Delta$ is related to the energy fluctuations as

$$
\Delta=C_{c}\left[\frac{\sigma^{2}(E)}{V_{0}^{2}}\right]^{1 / 2}
$$

where $\sigma^{2}(E)$ is the variance of the energies of the electrons and $C_{c}$ is coefficient of chromatic aberration the we can solve for the standard deviation of the energy:

$$
\sigma(E)=\left[V_{0}^{2} \Delta^{2} / C_{c}^{2}\right]^{1 / 2}
$$

which with the $C_{c}=1 \mathrm{~mm}$ calculated for the polepiece of the instrument corresponds to an $0.3 \mathrm{eV}$ energy spread, just as expected from the width of the zeroloss peak as measured by electron energy loss spectroscopy (EELS).

\section{Comparison of bright field detector configura- tions}

The conventional STEM bright field detector consists of a small circular detector placed in the middle of the bright field disk as shown in Figure 5a. The detector must be about an order of magnitude smaller than the convergence angle of the probe in order to maintain sufficient coherence to be equivalent via reciprocity to phase contrast imaging in conventional high resolution transmission electron microscopy (HRTEM). This restriction means that the conventional bright field (BF) detector makes use of a very small proportion of the area of the bright field disk. A correspondingly small fraction of the incident electrons are used to from the conventional BF signal.

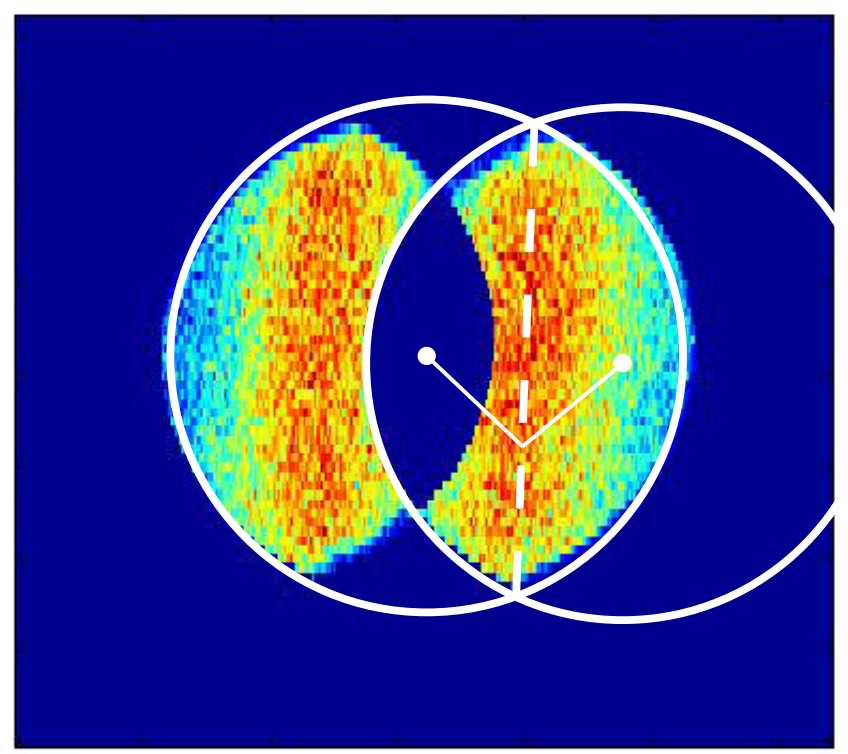

Figure 4: Illustration of how the achromatic lines arise in the amplitude of $G\left(\mathbf{K}_{f}, \mathbf{Q}_{p}\right)$ at points equidistant from the centres of the zero order and scattered disks. The white circles indicate the approximate positions of the zero order and a scattered disks. The centres of the disks are indicated by the small solid dots and can be seen to be equidistant from the achromatic line, indicated by the dashed line, superimposed over experimental data (the same as in Figure 2 , in the double overlap regions.
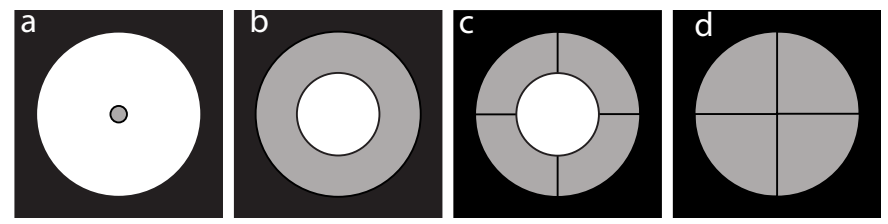

Figure 5: Schematic showing the geometries of a conventional BF detector (A), an $\mathrm{ABF}$ detector (B), the quadrant detectors used in DPC, annular (C) and full quadrants (D). The convergence angle is indicated by the inner bound of the black outer region. Detectors are coloured grey with black edges. 


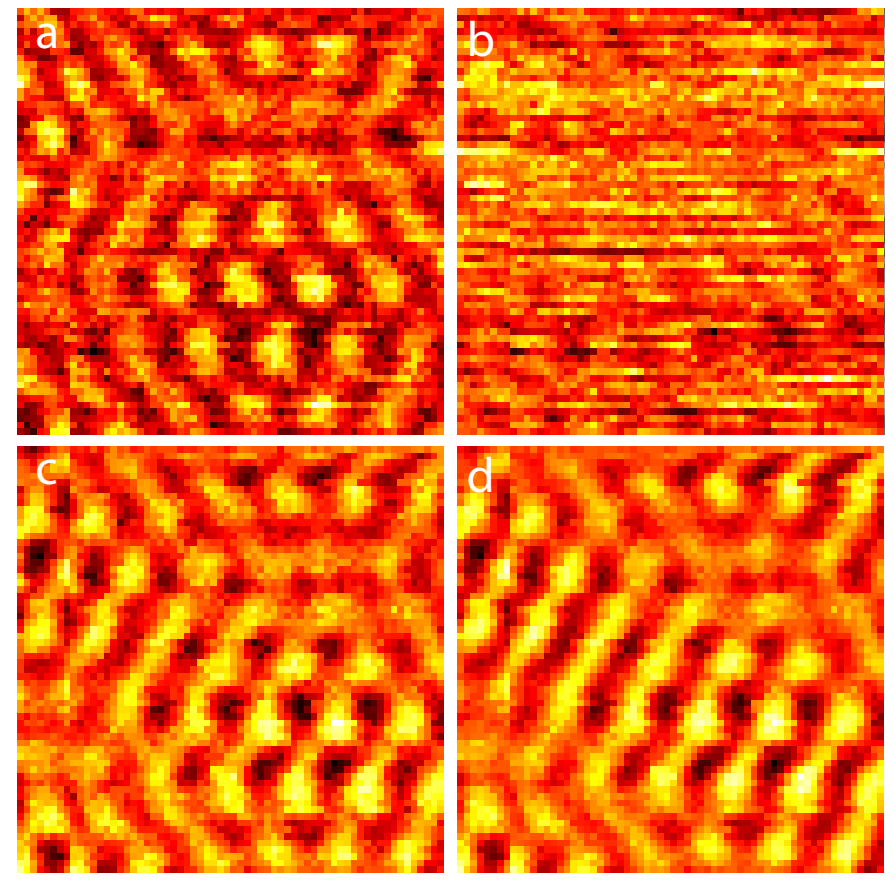

Figure 6: Conventional BF (a), ABF (b), annular DPC (c) and DPC (d) images of bilayer graphene. The images were created from the same dataset as the high efficiency ptychography phase contrast image in Figure 3 As seen here, ABF shows very little contrast for weak phase objects.

There has been a recent resurgence of interest in the $\mathrm{ABF}$ mode of imaging (Figure 5b) for atomic resolution imaging of light elements in aberration corrected STEM [10. The method has gained popularity as it is capable of revealing columns of low atomic number even when neighbouring columns of high atomic number, which can be difficult with $\mathrm{ADF}$ imaging. Although the method is less sensitive to defocus and thickness compared to conventional BF imaging, some complications in interpretability remain. $\mathrm{ABF}$ image contrast may be generated through channelling effects, or through phase contrast effects if residual aberrations are present.

DPC has for many years been used for imaging magnetic fields [14, 15] and recently atomic resolution mapping of electrostatic fields has been demonstrated [16. In DPC the bright field disk is segmented into multiple detectors, typically in quadrants. DPC can be performed using annular quadrants such as in Figure 50 or using the entire bright field disk as in Figure 5d.

Figure 6 displays conventional BF, ABF, and DPC images constructed from the same $4 \mathrm{D}$ dataset as the phase contrast image formed using ptychography shown in Figure 3 As expected, ptychography provides the clearest image of the bilayer graphene. DPC produces the second highest signal to noise ratio. The DPC images are are however differential images, and the atomic columns are located at the inflection points between high and low intensity rather than at the intensity extrema. DPC using full quadrants produces slightly less noisy images than
DPC using annular quadrants. Although the conventional $\mathrm{BF}$ image appears more noisy than the DPC images, it is still a far higher quality image than that produced by $\mathrm{ABF}$, which shows almost no lattice contrast at all.

The lack of contrast in the ABF image results from the lack of significant channelling with such a thin sample and because the aberration corrector has been tuned to compensate for aberrations up to fifth order, leaving only small residual aberrations. In the absence of aberrations the phase of the wavefunction transferred at $\mathbf{K}_{f}$ will be of equal amplitude but of opposite phase to that transmitted at $-\mathbf{K}_{f}$ due to equation 3 for a given spatial frequency $\mathbf{Q}_{p}$. We can examine the image produced by a centrosymmetric detector by considering the sum

$$
\begin{aligned}
G\left(\mathbf{K}_{f}, \mathbf{Q}_{p}\right)+ & G\left(-\mathbf{K}_{f}, \mathbf{Q}_{p}\right) \\
= & \left|A\left(\mathbf{K}_{f}\right)\right|^{2} \delta\left(\mathbf{Q}_{p}\right)+\left|A\left(-\mathbf{K}_{f}\right)\right|^{2} \delta\left(\mathbf{Q}_{p}\right) \\
& +A\left(\mathbf{K}_{f}\right) A^{*}\left(\mathbf{K}_{f}+\mathbf{Q}_{p}\right) \Psi_{s}^{*}\left(-\mathbf{Q}_{p}\right) \\
& +A\left(-\mathbf{K}_{f}\right) A^{*}\left(-\mathbf{K}_{f}+\mathbf{Q}_{p}\right) \Psi_{s}^{*}\left(-\mathbf{Q}_{p}\right) \\
& +A^{*}\left(\mathbf{K}_{f}\right) A\left(\mathbf{K}_{f}-\mathbf{Q}_{p}\right) \Psi_{s}\left(\mathbf{Q}_{p}\right) \\
& +A^{*}\left(-\mathbf{K}_{f}\right) A\left(-\mathbf{K}_{f}-\mathbf{Q}_{p}\right) \Psi_{s}\left(\mathbf{Q}_{p}\right) .
\end{aligned}
$$

In the absence of aberrations, and for a radially symmetric objective aperture, the complex conjugates are irrelevant and can be ignored and

$$
\begin{aligned}
& A^{*}\left(\mathbf{K}_{f}+\mathbf{Q}_{p}\right)=A\left(-\mathbf{K}_{f}-\mathbf{Q}_{p}\right), \\
& A^{*}\left(-\mathbf{K}_{f}+\mathbf{Q}_{p}\right)=A\left(\mathbf{K}_{f}-\mathbf{Q}_{p}\right),
\end{aligned}
$$

and

$$
A\left(\mathbf{K}_{f}\right)=A\left(-\mathbf{K}_{f}\right)
$$

so

$$
\begin{aligned}
G\left(\mathbf{K}_{f}, \mathbf{Q}_{p}\right)+ & G\left(-\mathbf{K}_{f}, \mathbf{Q}_{p}\right) \\
= & 2\left|A\left(\mathbf{K}_{f}\right)\right|^{2} \delta\left(\mathbf{Q}_{p}\right) \\
& +\left[\Psi_{s}^{*}\left(-\mathbf{Q}_{p}\right)+\Psi_{s}\left(\mathbf{Q}_{p}\right)\right] \\
& \times\left[A\left(-\mathbf{K}_{f}\right) A^{*}\left(-\mathbf{K}_{f}+\mathbf{Q}_{p}\right)\right. \\
& \left.+A\left(\mathbf{K}_{f}\right) A^{*}\left(\mathbf{K}_{f}+\mathbf{Q}_{p}\right)\right] .
\end{aligned}
$$

However, from equation 3

$$
\Psi_{s}^{*}\left(-\mathbf{Q}_{p}\right)+\Psi_{s}\left(\mathbf{Q}_{p}\right)=0
$$

and so

$$
\begin{aligned}
G\left(\mathbf{K}_{f}, \mathbf{Q}_{p}\right) & +G\left(-\mathbf{K}_{f}, \mathbf{Q}_{p}\right) \\
& =2\left|A\left(\mathbf{K}_{f}\right)\right|^{2} \delta\left(\mathbf{Q}_{p}\right) .
\end{aligned}
$$

This implies that in the absence of aberrations any centrosymmetric bright field detector will give zero contrast for a weak phase object. Essentially ABF sums the double overlap regions which are $\pi$ out of phase, cancelling any transfer of the wavefunctions in the absence of aberrations 

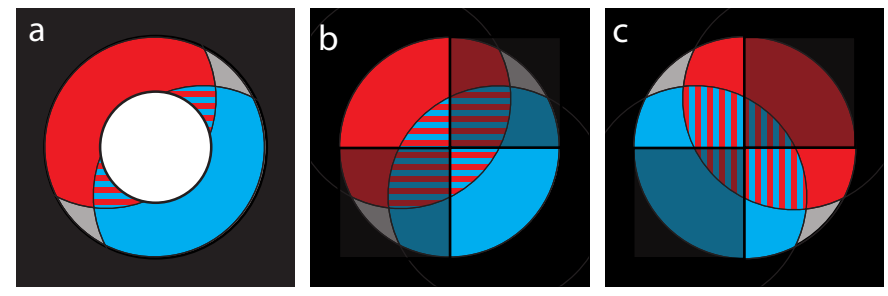

Figure 7: Schematic showing how the $\mathrm{ABF}$ (a) and DPC (b and c) detector geometries interact with $G\left(\mathbf{K}_{f}, \mathbf{Q}_{p}\right)$. Regions of double and triple overlap are indicated for a fixed $\mathbf{Q}_{p}$ as in Figure 1 ABF sums regions of double overlap which transfer out of phase but with equal amplitude and hence cancel. (b) For the same spatial frequency as in (a), a DPC image created by subtracting the upper left quadrant from the lower right quadrant will effectively bring the two double overlap regions into phase, so that they add rather than cancel. However, for other spatial frequencies the subtraction does not alleviate the cancellation. In (c) equal amounts of both out of phase double overlap regions exist over each of the two quadrants, and therefore cancel out on both quadrants.

and channelling as illustrated in Figure $7 \mathrm{~h}$. Although materials such as graphene contain very light elements, ABF imaging can provide only very poor contrast with such materials in a well tuned aberration corrected STEM.

Because opposite quadrants are subtracted in DPC it does not suffer so severely from the cancellation of the wavefunctions transferred in opposing double overlap regions. For example, in Figure 7b subtracting the top left quadrant from the bottom right quadrant reverses the effect of the $\pi$ phase difference and the wavefunctions for this spatial frequency transfer, rather than cancelling as in ABF. However, because the position of the double overlap regions changes with spatial frequency, quadrants that work well for one spatial frequency will not work so well for others. If the double overlap regions shown in Figure $7 \mathrm{~b}$ are rotated, there will be an increasing amount of both of the blue and red double overlap regions on each of these quadrants, until the situation shown in Figure $7 \mathrm{~F}$ is reached, in which equal amounts of the blue and red double overlap regions reside on both quadrants. For spatial frequencies where this occurs, the phase difference will cancel any wavefunction transfer and the signal from each quadrant will be zero. The amount a given spatial frequency is transferred in DPC therefore decreases in proportion to the amount the two double overlap regions share the same quadrants.

In contrast to DPC, with ptychography we are able to use the optimal region for every spatial frequency independently. By using all of the double overlap regions available for each spatial frequency we maximise the collection efficiency for all accessible spatial frequencies. Furthermore by setting the amplitude to zero outside the double overlap regions for all nonzero frequencies we reject efficiency reducing noise and achieve the maximum efficiency phase contrast imaging possible. This efficiency is evidenced in the clarity of Figure 3 a over those images shown in Figure 6.

\section{Conclusion}

The ronchigram contains a wealth of information which is currently left unutilised. In many cases, beam damage is now the major factor limiting STEM studies. As we have shown, by recording a ronchigram at every probe position, it is possible to construct a significantly higher efficiency phase contrast images through ptychography. With a fast enough camera it will be possible to harness the efficiency of the method and use rapid scanning to perform low dose imaging of beam sensitive materials. Gaining as much information as possible from each electron enables using the minimum possible dose. By using the entire bright field disk to perform phase contrast imaging at double the conventional bright field resolution in combination with ADF imaging we essentially use all available angles and make use of all useful transmitted electrons.

The phase contrast images produced with the method should also be sensitive to local electromagnetic fields, as with DPC, but with greater sensitivity. This sensitivity should also make the technique of great interest to fields such as magnetic and ferroelectric materials. The four dimensional dataset also offers the ability to access additional information about the microscope itself. As we have illustrated, the effects of chromatic aberration can be diagnosed. Furthermore it should be possible to diagnose the residual aberrations on crystals using ptychography. With the ever increasing speed of pixelated detectors, we foresee the widespread adoption of their use in STEM to extract four dimensional data sets such as described herein.

\section{Acknowledgements}

This research was supported by the EPSRC through the UK National Facility for Aberration-Corrected STEM (SuperSTEM) and also EPSRC grant number EP/K032518/1. Research at Oak Ridge National Laboratory was sponsored by the Division of Materials Sciences and Engineering of the U.S. department of Energy. The work was partially supported by the European Union Seventh Framework Programme under Grant Agreement 312483 - ESTEEM2 (Integrated Infrastructure InitiativeI3).

\section{References}

\section{References}

[1] R. Henderson, The Potential And Limitations of Neutrons, Electrons And X-Rays For Atomic-Resolution Microscopy of Unstained Biological Molecules, Quarterly Reviews of Biophysics 28 (2) (1995) 171-193.

[2] O. Scherzer, The Theoretical Resolution Limit of the Electron Microscope, Journal of Applied Physics 20 (1) (1949) 20.

[3] K. W. Urban, C. L. Jia, L. Houben, M. Lentzen, S. B. Mi, K. Tillmann, Negative spherical aberration ultrahigh-resolution imaging in corrected transmission electron microscopy, Philos T R Soc A 367 (1903) (2009) 3735-3753.

[4] R. M. Glaeser, Invited Review Article: Methods for imaging weak-phase objects in electron microscopy, Review of Scientific Instruments 84 (11) (2013) 111101. 
[5] J. M. Cowley, Image Contrast in a Transmission Scanning Electron Microscope, Appl. Phys. Lett. 15 (2) (1969) 58.

[6] E. Zeitler, M. G. R. Thomson, Scanning Transmission Electron Microscopy 1, Optik 31 (3) (1970) 258.

[7] E. Zeitler, M. G. R. Thomson, Scanning Transmission Electron Microscopy 2, Optik 31 (4) (1970) 359.

[8] H. Rose, Phase-contrast in Scanning-Transmission ElectronMicroscopy, Optik 39 (4) (1974) 416.

[9] R. Ishikawa, E. Okunishi, H. Sawada, Y. Kondo, F. Hosokawa, E. Abe, Direct imaging of hydrogen-atom columns in a crystal by annular bright-field electron microscopy, Nat Mater 10 (4) (2011) 278-281.

[10] S. Findlay, N. Shibata, H. Sawada, E. Okunishi, Dynamics of annular bright field imaging in scanning transmission electron microscopy, Ultramicroscopy.

[11] N. H. Dekkers, H. D. Lang, Differential Phase Contrast in a STEM, Optik 41 (4) (1974) 452-456.

[12] H. Rose, Image-formation by Inelastically Scattered Electrons in Electron-Microscopy, Optik 45 (2) (1976) 139-158.

[13] H. Rose, Nonstandard imaging methods in electron microscopy, Ultramicroscopy 2 (1977) 251-267.

[14] J. N. Chapman, P. E. Batson, E. M. Waddell, R. P. Ferrier, The direct determination of magnetic domain wall profiles by differential phase contrast electron microscopy, Ultramicroscopy 3 (1978) 203-214.

[15] J. N. Chapman, I. R. McFadyen, S. McVitie, Modified differential phase contrast Lorentz microscopy for improved imaging of magnetic structures, IEEE Trans. Magn. 26 (5) (1990) 15061511.

[16] N. Shibata, S. D. Findlay, Y. Kohno, H. Sawada, Y. Kondo, Y. Ikuhara, Differential phase-contrast microscopy at atomic resolution, Nat Mater 8 (8) (2012) 611-615.

[17] J. M. Rodenburg, B. C. McCallum, P. D. Nellist, Experimental tests on double-resolution coherent imaging via STEM, Ultramicroscopy 48 (3) (1993) 304-314.

[18] P. D. Nellist, B. C. McCallum, J. M. Rodenburg, Resolution beyond the 'information limit' in transmission electron microscopy, Nature 374 (1995) 630.

[19] F. Hüe, J. M. Rodenburg, A. M. Maiden, F. Sweeney, P. A. Midgley, Wave-front phase retrieval in transmission electron microscopy via ptychography, Phys Rev B 82 (2010) 121415

[20] M. J. Humphry, B. Kraus, A. C. Hurst, A. M. Maiden, J. M. Rodenburg, Ptychographic electron microscopy using high-angle dark-field scattering for sub-nanometre resolution imaging, Nat Comms 3 (2012) 730.

[21] T. M. Godden, R. Suman, M. J. Humphry, J. M. Rodenburg, A. M. Maiden, Ptychographic microscope for three-dimensional imaging, Opt. Express 22 (10) (2014) 12513.

[22] D. J. Batey, D. Claus, J. M. Rodenburg, Information multiplexing in ptychography, Ultramicroscopy 138 (2014) 13-21.

23] F. Berenguer, R. J. Bean, L. Bozec, J. Vila-Comamala, F. Zhang, C. M. Kewish, O. Bunk, J. M. Rodenburg, I. K. Robinson, Coherent X-Ray Imaging of Collagen Fibril Distributions within Intact Tendons, Biophysical Journal 106 (2) (2014) 459-466.

[24] F. Zhang, I. Peterson, J. Vila-Comamala, A. Diaz, F. Berenguer, R. Bean, B. Chen, A. Menzel, I. K. Robinson, J. M. Rodenburg, Translation position determination in ptychographic coherent diffraction imaging, Opt. Express 21 (11) (2013) 13592

[25] A. M. Maiden, G. R. Morrison, B. Kaulich, A. Gianoncelli, J. M. Rodenburg, Soft X-ray spectromicroscopy using ptychography with randomly phased illumination, Nat Comms 4 (2013) 1669.

[26] O. L. Krivanek, J. P. Ursin, N. J. Bacon, G. J. Corbin, N. Dellby, P. Hrncirik, M. F. Murfitt, C. S. Own, Z. S. Szilagyi, High-energy-resolution monochromator for aberrationcorrected scanning transmission electron microscopy/electron energy-loss spectroscopy, Philos T R Soc A 367 (1903) (2009) 3683-3697.

[27] P. D. Nellist, J. M. Rodenburg, Beyond the conventional information limit: the relevant coherence function, Ultramicroscopy
54 (1) (1994) 61-74.

[28] N. Dellby, N. J. Bacon, P. Hrncirik, M. F. Murfitt, G. S. Skone, Z. S. Szilagyi, O. L. Krivanek, Dedicated STEM for 200 to 40 keV operation, Eur Phys J-Appl Phys 54 (3) (2011) 33505.

[29] R. Ishikawa, A. R. Lupini, S. D. Findlay, S. J. Pennycook, Quantitative Annular Dark Field Electron Microscopy Using Single Electron Signals, Microscopy and Microanalysis 20 (01) (2013) 99-110.

[30] B. Kabius, P. Hartel, M. Haider, H. Müller, S. Uhlemann, U. Loebau, J. Zach, H. Rose, First application of Cc-corrected imaging for high-resolution and energy-filtered TEM, Journal of Electron Microscopy 58 (3) (2009) 147-155. 